\title{
Iter diagnostico nel paziente con iperparatiroidismo primario recidivato dopo paratiroidectomia
}

\author{
Federica Saponaro ${ }^{1} \cdot$ Filomena Cetani $^{1}$
}

Accettato: 11 febbraio 2020 / Pubblicato online: 4 settembre 2020

(c) The Author(s) 2020

\section{Introduzione}

L'iperparatiroidismo primario (IP) è la terza endocrinopatia più diffusa ed è caratterizzato da ipercalcemia e livelli di PTH aumentati o inappropriatamente normali. Le complicanze cliniche della malattia sono legate prevalentemente a un coinvolgimento osseo e renale, sintomi non classici sono dati dal coinvolgimento neuropsichico e cardiovascolare. La terapia è chirurgica (paratiroidectomia, PTx) per tutti i pazienti con IP sintomatico e per i pazienti con IP asintomatico, secondo precisi criteri proposti dalle linee di consenso internazionali [1]. Il successo della PTx si riflette nella normalizzazione dei livelli di calcio e PTH in modo duraturo, con una percentuale di guarigione fino al $98 \%$ (nei centri a elevato volume di PTx), con effetti positivi sulle complicanze della malattia [2].

Raramente si verifica una persistenza di malattia, quando i livelli di calcio sierico e PTH rimangono elevati dopo la PTx o una recidiva, quando l'ipercalcemia ricompare dopo almeno 6 mesi di eucalcemia, dal momento dalla PTx. Le cause di persistenza/recidiva ( $\mathrm{p} / \mathrm{r}$ ) sono: 1) la mancata localizzazione e asportazione dell' adenoma paratiroideo; 2) un'inadeguata resezione in pazienti con malattia multighiandolare sincrona; 3) la successiva comparsa di un ulteriore adenoma in pazienti con malattia multighiandolare asincrona; 4) la presenza di un secondo adenoma occulto in sede eutopica o ectopica; o 5) la persistenza di tessuto paratiroideo iperfunzionante nei rari casi di paratireosi o carcinoma delle paratiroidi [3].

F. Saponaro

federica.saponaro@unipi.it

1 Endocrinologia 2, Dipartimento di Medicina Clinica e Sperimentale, Università degli Sudi di Pisa, Pisa, Italia

\section{Diagnosi clinica, biochimica e molecolare}

In tutti i pazienti con sospetta $\mathrm{p} / \mathrm{r}$ è obbligatorio confermare la diagnosi ed effettuare un'accurata anamnesi familiare. Infatti, per la stessa natura genetica della condizione e il frequente coinvolgimento di più ghiandole, $1 \mathrm{la} / \mathrm{r}$ si verifica più spesso nelle forme familiari di IP (Sindrome delle neoplasie endocrine multiple tipo 1, MEN1, tipo 2A, MEN2A, tipo 4, MEN4, Sindrome dell'iperparatiroidismo associato ai tumori della mandibola/mascella, iperparatiroidismo familiare isolato). La conferma della diagnosi prevede l'esclusione di terapie in atto che possano alterare il metabolismo fosfo-calcico (diuretici tiazidici e litio) e di forme secondarie di iperparatiroidismo da insufficienza renale cronica, malassorbimento gastrointestinale o deficit di vitamina D. Inoltre, sarà necessaria una revisione critica della diagnosi preoperatoria, per confermarne la correttezza [4].

Le indagini biochimiche da effettuare comprendono il dosaggio del calcio totale e corretto per albumina, del calcio ionizzato (laddove disponibile), del PTH intatto, della $25 \mathrm{OH}$-vitamina $\mathrm{D}$ e del calcio urinario delle 24 ore. Un rapporto della clearance del calcio/creatinina $<0,01$ è suggestivo di ipercalcemia ipocalciurica familiare $(\mathrm{FHH})$, una rara ma benigna condizione che mima l'IP, dovuta a una mutazione inattivante del recettore del calcio (FHH tipo 1), di GNA11 (FHH di tipo 2) o di AP2S1 (FHH di tipo $3)$ che non richiede in genere l'intervento chirurgico. Nel sospetto di una forma sindromica di IP è opportuno eseguire una valutazione funzionale e morfologica ipofisaria e del tratto gastroenteropancreatico e l'ortopantomografia. Nei casi di $\mathrm{p} / \mathrm{r}$ da malattia multighiandolare è consigliabile eseguire il test genetico dei geni responsabile della MEN1 (MEN1), MEN4 (CDKN1B) e IP a associato ai tumori della mandibola/mascella (CDC73) [5]. 


\section{Indicazioni al reintervento}

Le indicazioni per il reintervento sono le stesse applicabili per la prima PTx, con alcune importanti considerazioni legate alla maggiore difficoltà e insorgenza di complicanze. Di primaria importanza in questi casi è affidare il paziente a un team esperto in ambito di patologia delle paratiroidi e, soprattutto, a un chirurgo esperto. Se l'intervento di PTx già di per sé ha una ridotta probabilità curativa in mani poco esperte, ancora di più il reintervento pone sfide tecniche legate alla cicatrice, all'alterata anatomia del collo dopo la precedente PTx e alla maggiore difficoltà di preservare il nervo laringeo ricorrente. A questo proposito, una valutazione della motilità delle corde vocali prima di un reintervento è altamente consigliata, poiché la presenza di pregresse disfunzioni delle corde vocali può avere una ricaduta sulla decisione del reintervento.

Nel caso di p/r i pazienti con chiari sintomi renali e/o ossei saranno inviati sicuramente alla ri-esplorazione. Tuttavia, i pazienti con una diagnosi incerta oppure una malattia lieve/asintomatica potranno essere valutati con più cautela $\mathrm{e}$ seguiti con un'attenta sorveglianza.

\section{Imaging}

Nel caso di un reintervento per $\mathrm{p} / \mathrm{r}$ di IP, l'imaging ha un ruolo molto importante per una corretta localizzazione del tessuto paratiroideo persistente o recidivato. L'ecografia e la scintigrafia con 99-Tecnezio-Sestamibi, possibilmente in combinazione con la TC (SPECT/CT) offrono nel complesso una sensibilità di circa 1 ' $80 \%$. La sensibilità può variare tra i diversi centri sulla base della propria esperienza. La TC4D si è dimostrata in studi recenti una tecnica con una sensibilità e specificità superiore alla SPECT/CT. La RM può essere utilizzata in alcuni casi selezionati. Molto promettente è la recente metodica della PET/TC con ${ }^{18}$-Ffluorocolina, che ha dimostrato elevata sensibilità nell'identificazione di tessuto paratiroideo, quando l'imaging tradizionale risulti negativo. Infine, in centri specializzati è possibile accedere a tecniche di localizzazione intraoperatoria d'avanguardia come l' angiografia Intraoperative Indocyanine Green Angiografy, che consente di localizzare il tessuto paratiroideo iperfunzionante, che abbia captato il tracciante fluorescente [6].

Il tipo di approccio chirurgico è rappresentato generalmente dall'esplorazione del collo ma, in casi selezionati che presentino un imaging preoperatorio convincente e concordante, può essere utilizzato l'approccio chirurgico mininvasivo. Uno strumento essenziale è il dosaggio del PTH intraoperatorio: infatti, un inadeguato calo del PTH dopo l'escissione del tessuto paratiroideo rappresenta una chiara indicazione per il chirurgo a proseguire l'esplorazione. Il so- spetto di paratiroidi in sede ectopica richiederà l'esplorazione mediastinica che, nella grande maggioranza dei casi, può essere effettuata con un approccio chirurgico cervicale, che consente l'accesso sia alla parte anteriore che posteriore del mediastino [1].

\section{Conclusioni}

Per i pazienti che non possono essere sottoposti al reintervento, l'unica opzione possibile è la terapia medica. Questa include un' adeguata idratazione nei casi di ipercalcemia lieve e l'utilizzo di calciomimetici come il cinacalcet nei casi di ipercalcemia (calcio $>1 \mathrm{mg}$ al limite superiore del range della norma) in accordo con le linee guida EMA [7, 8], al fine di ottenere un controllo dell'ipercalcemia. I calciomimetici, tuttavia, non hanno dimostrato effetti positivi sugli organi bersaglio dell'IP e, in primis, sull'osso. Per il trattamento dell'osteoporosi, qualora questa sia presente, si potrà utilizzare la specifica terapia scegliendo i bisfosfonati come prima linea terapeutica.

Funding Note Open access funding provided by Università di Pisa within the CRUI-CARE Agreement.

Conflitto di interesse Le autrici Federica Saponaro e Filomena Cetani dichiarano di non avere conflitti di interesse.

Consenso informato Lo studio presentato in questo articolo non ha richiesto sperimentazione umana.

Studi sugli animali Le autrici di questo articolo non hanno eseguito studi sugli animali.

Nota della casa editrice Springer Nature rimane neutrale in riguardo alle rivendicazioni giurisdizionali nelle mappe pubblicate e nelle affiliazioni istituzionali.

Open Access This article is licensed under a Creative Commons Attribution 4.0 International License, which permits use, sharing, adaptation, distribution and reproduction in any medium or format, as long as you give appropriate credit to the original author(s) and the source, provide a link to the Creative Commons licence, and indicate if changes were made. The images or other third party material in this article are included in the article's Creative Commons licence, unless indicated otherwise in a credit line to the material. If material is not included in the article's Creative Commons licence and your intended use is not permitted by statutory regulation or exceeds the permitted use, you will need to obtain permission directly from the copyright holder. To view a copy of this licence, visit http://creativecommons.org/licenses/by/4.0/.

\section{Bibliografia}

1. Bilezikian JP, Brandi ML, Eastell R et al (2014) Guidelines for the management of asymptomatic primary hyperparathyroidism: summary statement from the fourth international workshop. J Clin Endocrinol Metab 99:3561-3569 
2. Saponaro F, Cetani F, Repaci A et al (2018) Clinical presentation and management of patients with primary hyperparathyroidism in Italy. J Endocrinol Invest 41(11):1339-1348

3. Udelsman R, Akerström G, Biagini C et al (2014) The surgical management of asymptomatic primary hyperparathyroidism: proceedings of the fourth international workshop. J Clin Endocrinol Metab 99:3595-3606

4. Udelsman R (2011) Approach to the patient with persistent or recurrent primary hyperparathyroidism. J Clin Endocrinol Metab 96(10):2950-2958

5. Borsari S, Pardi E, Pellegata NS et al (2016) Loss of p27 expression is associated with MEN1 gene mutations in sporadic parathyroid adenomas. Endocrine 2016:1-12
6. Spartalis E, Ntokos G, Georgiou K et al (2020) Intraoperative indocyanine green (ICG) angiography for the identification of the parathyroid glands: current evidence and future perspectives. In Vivo 34(1):23-32

7. Saponaro F, Faggiano A, Grimaldi F et al (2013) Cinacalcet in the management of primary hyperparathyroidism: post marketing experience of an Italian multicentre group. Clin Endocrinol (Oxf) 79:20-26

8. https://www.ema.europa.eu/en/medicines/human/EPAR/mimpara. Accessed on 03 September 2020 